\title{
Inflammatory marker alteration in response to systemic therapies in psoriasis
}

\author{
CRISTINA GRECHIN ${ }^{1}$, LAURA GHEUCĂ SOLOVĂSTRU ${ }^{2,3}$, DAN VÂȚA ${ }^{2,3}$, ADRIANA IONELA PĂTRAȘCU ${ }^{3}$, \\ ALINA IOANA GRĂJDEANU ${ }^{2}$ and ELENA PORUMB-ANDRESE ${ }^{2,3}$ \\ ${ }^{1}$ Department of Gastroenterology, Sligo University Hospital, Sligo, F91 H684, Ireland; \\ ${ }^{2}$ Department of Dermatology, 'Gr. T. Popa' University of Medicine and Pharmacy, 700115 Iasi; \\ ${ }^{3}$ Department of Dermatology, 'Sf. Spiridon' Clinical Emergency County Hospital, 700111 Iasi, Romania
}

Received December 9, 2019; Accepted January 13, 2020

DOI: $10.3892 / \mathrm{etm} .2020 .8535$

\begin{abstract}
Substantial research has focused on the presence of biomarkers involved in both the pathogenesis of psoriasis and its comorbidities. The identification of these biomarkers has a crucial role in establishing the diagnosis and prognosis, in understanding the physiopathological mechanism and in determining the therapeutic response. The aim of this study was to emphasize the alteration in inflammatory markers in response to systemic therapies in psoriasis. Evolution of inflammatory marker alteration was studied in 194 patients with psoriasis, aged between 7 and 87 years. Two groups were set up: the first comprised of patients treated with methotrexate $(n=51)$, while the second comprised patients treated with biological therapy $(n=143)$. Each group was evaluated for blood values of C-reactive protein (CRP), erythrocyte sedimentation rate (ESR) and fibrinogen before and after treatment, the fluctuation of these values according to the treatment, the interrelation between inflammatory markers and inflammatory activity of the disease and the evolution of the disease after treatment. In group I, 46 out of 51 patients had elevated levels of acute phase reactants before treatment. After treatment with methotrexate $7.5 \mathrm{mg} /$ week, 12 out of 46 patients had elevated blood levels of ESR and 18 out of 46 patients of CRP and fibrinogen. Before treatment with biological therapy, 138 patients out of 143 presented abnormal high range for acute phase reactants. After treatment with biological therapy, 18 patients out of 138 had elevated blood levels of ESR and 37 patients out of 138 had elevated CRP and fibrinogen. A favorable evolution was noted in 98 patients out of 138. It was concluded that the systemic treatment with both methotrexate and biological therapy showed a marked decline in the patients
\end{abstract}

Correspondence to: Dr Laura Gheucă Solovăstru, Department of Dermatology, 'Gr. T. Popa' University of Medicine and Pharmacy, Str. Universitatii 16, 700115 Iasi, Romania

E-mail: 1solovastru13@yahoo.com

Key words: inflammatory markers, psoriasis, biological therapy, immune dysregulation, genetic predisposition with abnormal values of CRP, ESR and fibrinogen, indirectly showing a decline in the inflammatory activity of psoriasis.

\section{Introduction}

Psoriasis is a chronic relapsing autoimmune disease, in which genetic and epigenetic changes result in an altered immune function, keratinocyte activation and hyperproliferation. It mainly affects the skin and joints (1). Some environmental factors, such as stress, are considered to lead to an immune dysregulation, but only in patients with genetic predisposition, resulting in an abnormal proliferation and maturation of keratinocytes, proliferation of dermal blood vessels, mast cell activation and skin infiltration by inflammatory cells (2-4). The inflammatory nature of psoriasis was illustrated by a systemic and dermal secretion of cytokines such as interleukins (IL): IL-2, IL-6, IL-8, IL-17, IL-18, IL-22, IL-23, IL-24, interferon- $\gamma$ (INF- $\gamma$ ) and tumor necrosis factor- $\alpha(\mathrm{TNF}-\alpha)(5)$. TNF- $\alpha$ is a cytokine secreted by lymphocyte $\mathrm{T}$, keratinocytes and dermal macrophages, $\mathrm{CD} 11^{+}$ dendritic cells and mastocytes (6). TNF- $\alpha$ increases the production of IL- 6 and ICAM-1 expression. IL-6 secretion induced by TNF- $\alpha$ leads to hepatic stimulation of production of acute phase reactants such as C-reactive protein (CRP) and fibrinogen (7). CRP is an acute phase protein that indirectly illustrates pro-inflammatory activity of cytokines. For this reason, CRP is considered to be an inflammatory marker (8). The study of Rocha-Pereira et al (9) presented the association between inflammation and psoriasis through increased levels of CRP, fibrinogen, haptoglobin, C3, C4 and the fact that the blood levels increase with the severity of the disease. They also proposed that blood values of elastase, CRP, elastase/ $\alpha$-macroglobulin and elastase/neutrophil ratios to be used for prognosis regarding the worsening of psoriasis. Gisondi et al (10) studied the correlation between high blood levels of CRP, resistin, chemerin and psoriasis before treatment and a reduction of these inflammatory marker levels after biological therapy. It is very important to monitor and control inflammation in order to chase-up the evolution of the disease and its comorbidities. It has been proved that IL- 6 induces type two diabetes mellitus and cardiovascular diseases and that INF- $\alpha$ could be involved in causing atherosclerosis (11). 
Devaraj et al (12) showed that CRP is a risk factor for cardiovascular disease, as well as a predictor factor for vascular events. They proposed treatment with statins for patients with CRP $>2 \mathrm{mg} / \mathrm{l}$ and metabolic syndrome.

The aim of the present study was to emphasize the role of the inflammatory syndrome in psoriasis [via CRP, fibrinogen, and erythrocyte sedimentation rate (ESR)], its evolution after treatment and its role in psoriasis comorbidities.

\section{Patients and methods}

This study included 194 patients with psoriasis aged between 7 and 83 years. It has been observed that psoriasis is frequent in middle-aged adults and elderly, in comparison to children and young adults, where there is a lower prevalence.

The 194 patients were treated differently, according to the severity of the disease: 51 patients with moderate psoriasis were treated with methotrexate (group I) and 143 patients with severe psoriasis were treated with biological therapy (group II).

In this study, the patients with moderate and severe psoriasis were selected defined by the extent of body surface area (BSA) involvement. This method was chosen because it is currently the preferred severity assessment instrument used in clinical practice. Using BSA, the patients were divided into three categories: Mild psoriasis $(<3 \% \mathrm{BSA})$, moderate psoriasis (3-10\% BSA) and severe psoriasis (>10\% BSA). The evolution of the inflammatory syndrome was followed via gradation of the blood levels for ESR, CRP and fibrinogen before and after treatment. Two groups of patients were established. Group I included 51 patients with moderate psoriasis treated with methotrexate $7.5 \mathrm{mg}$ /week. Group II included 143 patients with severe psoriasis treated with biological therapy according to the specific features of each patient: Etanerceptum (Enbrel) $50 \mathrm{mg}$ x $2 /$ week was administered subcutaneously x12 weeks, then $50 \mathrm{mg} /$ week $x 12$ weeks $(\mathrm{n}=51)$, infliximab (Remicade) $5 \mathrm{mg} / \mathrm{kg}$ intravenously $0,2,6$ week, then every 8 weeks $(\mathrm{n}=48)$, adalimumab (Humira) initial $80 \mathrm{mg}$, followed by $40 \mathrm{mg}$ subcutaneously every 2 weeks, starting one week after the initial dose $(\mathrm{n}=42)$, ustekinumab (Stelara) $45 \mathrm{mg}$ subcutaneously 0.4 week, then every 12 weeks $(n=2)$.

This study was approved by the Clinical Research Ethics Committee of 'Sf. Spiridon' Clinical Emergency County Hospital (Iasi, Romania) and the Ethics Committee of 'Gr. T. Popa' University of Medicine and Pharmacy (Iasi, Romania). Written informed consents were obtained by the patients and/or guardians.

\section{Results}

In group I, 46 out of 51 patients (90.2\%) had elevated levels of acute phase reactants before treatment. After treatment with methotrexate $7.5 \mathrm{mg} /$ week, 12 out of 51 patients $(23.5 \%)$ had elevated blood levels of ESR and 18 out of 51 patients had elevated CRP and fibrinogen (35.3\%) (Fig. 1).

Eighteen patients out of $51(35.3 \%)$ presented a favorable evolution, 22 patients had a slow favorable evolution, four patients had a fluctuant evolution (43.1\%) and seven patients had an unfavorable evolution (13.7\%) (Fig. 2).

A reduction of blood levels was observed in inflammatory markers after treatment with methotrexate and an

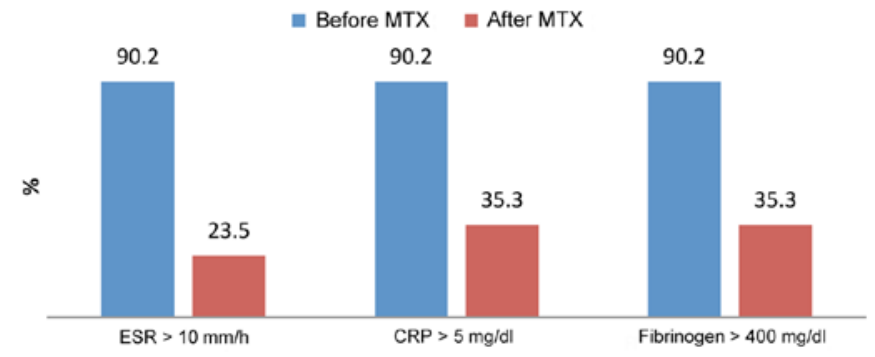

Figure 1. Progression of the inflammatory markers after treatment with methotrexate.

घavorable Slow favorable Eluctuant Unfavorable

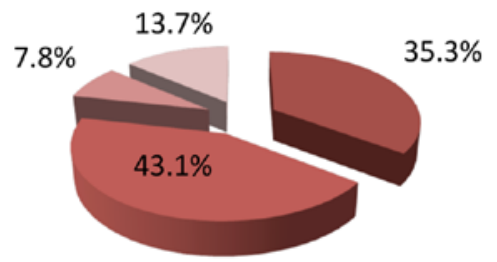

Figure 2. Distribution of patients according to the evolution of disease after treatment with methotrexate.

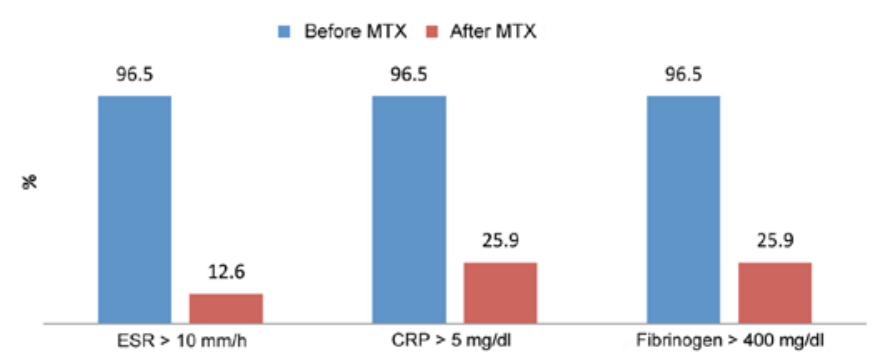

Figure 3. Progression of the inflammatory markers after treatment with biological therapy.

improvement of the well-being of patients-index measured by quality of life questionnaire (QOL). The clinical evolution was assessed using the Psoriasis Area Severity Index questionnaire and clinical symptoms (pruritus and pain, which are known to negatively impact the patients' lives).

Group II contained 143 patients with severe psoriasis treated with biological therapy. Before treatment, 138 patients out of 143 presented abnormal high range for acute phase reactants $(96.5 \%)$. After treatment, a dramatic decrease of acute phase reactants blood levels was observed. After treatment, 18 patients out of 138 had elevated blood levels of ESR $(12.6 \%)$ and 37 patients out of 138 elevated CRP and fibrinogen (25.9\%) (Fig. 3).

A favorable evolution was noticed in 98 patients out of $143(70 \%)$, a slow favorable evolution in 35 patients $(25 \%)$, a fluctuant evolution in only 3 patients $(2.9 \%)$ and an unfavorable evolution in 7 patients (2.1\%) (Fig. 4).

Biological therapy showed the best results and our results correlated with the scientific data that support the beneficial effect of biological therapy in decreasing inflammation in psoriasis (Table I and Figs. 5 and 6). 
Table I. Statistical analysis between the two groups of therapy.

\begin{tabular}{|c|c|c|c|c|c|c|c|c|}
\hline \multirow[b]{2}{*}{ Parameters } & \multicolumn{4}{|c|}{ Group I $(n=51)$} & \multicolumn{4}{|c|}{ Group II $(n=143)$} \\
\hline & $\begin{array}{c}\text { Before } \\
\text { treatment }\end{array}$ & $\begin{array}{c}\text { After } \\
\text { treatment }\end{array}$ & $\begin{array}{l}\text { P-value for paired } \\
\text { samples t-test }\end{array}$ & $\begin{array}{l}\text { Favorable } \\
\text { evolution }\end{array}$ & $\begin{array}{c}\text { Before } \\
\text { treatment }\end{array}$ & $\begin{array}{c}\text { After } \\
\text { treatment }\end{array}$ & $\begin{array}{l}\text { P-value for paired } \\
\text { samples t-test }\end{array}$ & $\begin{array}{l}\text { Favorable } \\
\text { evolution }\end{array}$ \\
\hline ESR & $0.92 \pm 0.13$ & $0.33 \pm 0.25$ & 0.001 & $26.1 \%{ }^{\mathrm{a}}$ & $1.17 \pm 1.19$ & $0.59 \pm 0.16$ & 0.001 & $13.0 \%$ \\
\hline CRP & $476 \pm 20.4$ & $365 \pm 54.4$ & 0.174 & $39.1 \%$ & $485 \pm 38.6$ & $363 \pm 76.5$ & 0.001 & $26.8 \%$ \\
\hline Fibrinogen & $20.7 \pm 2.89$ & $8.67 \pm 8.14$ & 0.045 & $39.1 \%$ & $18.8 \pm 3.04$ & $6.10 \pm 5.05$ & 0.001 & $26.8 \%$ \\
\hline
\end{tabular}

${ }^{\mathrm{a}} \mathrm{P}=0.039$ for $\chi^{2}$ test Lot I vs. Lot II. ESR, erythrocyte sedimentation rate; CRP, C-reactive protein.

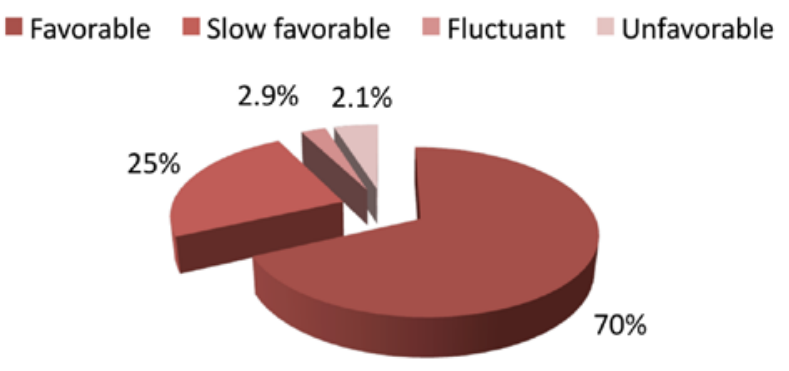

Figure 4. Distribution of the patients according to the evolution of disease after treatment with biological therapy.

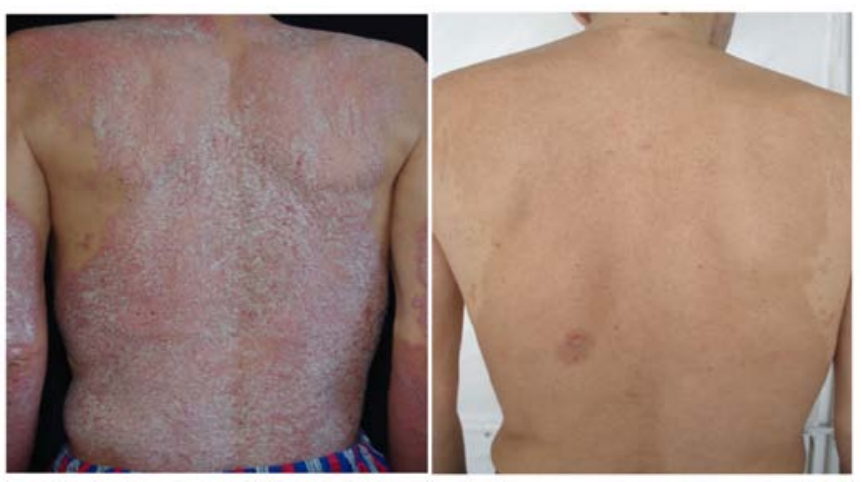

Figure 5. Male patient with psoriasis before and after treatment with biological therapy.

\section{Discussion}

This study sought to assess blood levels for CRP, ESR and fibrinogen before and after treatment with methotrexate and biological therapy, the respective blood level fluctuation according to the treatment, the association between CRP, ESR, fibrinogen and inflammatory activity of psoriasis and the evolution of the disease after treatment. There are different reports illustrating that blood levels of CRP, ESR and fibrinogen are indirectly related to the inflammatory activity of TNF- $\alpha$ and other cytokines, such as IL-6 that lead to hepatic stimulation of production for acute phase reactants such as CRP and fibrinogen (11). Biljan et al (13) and Isha et al (14) also observed that high levels of CRP are correlated with inflammatory activity of psoriasis.

After treatment with methotrexate and biological therapy, an important decrease in blood levels of CRP, ESR, and

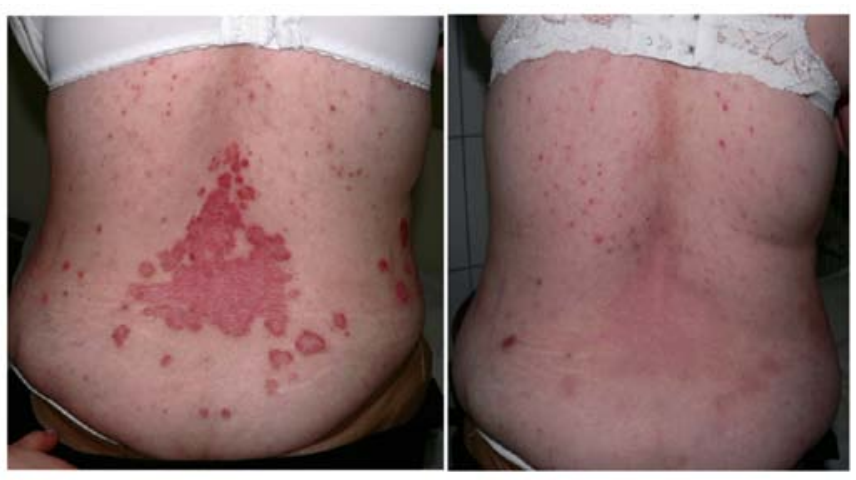

Figure 6. Female patient with psoriasis before and after treatment with biological therapy.

fibrinogen was observed leading to reduction of the inflammatory activity of the psoriasis.

After treatment with methotrexate, the inflammatory markers were reduced in 34 patients: From the initial number of 46 patients with ESR high levels to 12, and in 28 patients from the initial number of 46 patients with CRP and fibrinogen with elevated blood levels to 18 patients. Methotrexate inhibits dihydrofolate reductase, leading to a blockade of purines and pyrimidines synthesis, hence the DNA. Methotrexate inhibits AICAR transformylase as well, leading to an accumulation of adenosine with a strong pro inflammatory effect (15). When used long-term, methotrexate can cause side effects, such as pancytopenia, megaloblastic anaemia, nausea, vomiting, hepatic fibrosis, pulmonary fibrosis, allergies and foetal malformation (16).

Biological therapy showed the best results. Eighteen patients out of 138 for ESR and 37 patients out of 138 for CRP and fibrinogen presented elevated blood levels after treatment.

Zamanian et al (17) reported a decline of ESR and CRP levels after treatment with infliximab. Strober et al (18) illustrated that patients with psoriasis have a significant systemic inflammation trough high CRP levels. They observed that after Etanercept treatment, the CRP levels plummeted. This therapy is superior compared to others, but not all patients could be treated with it because of the selection criteria that had to be strictly followed. Kanelleas et al (19) studied 41 psoriasis patients treated with etanercept. They concluded that CRP, fibrinogen and ESR in association with PASI score show inflammatory status in psoriasis. They purposed these 
three markers to be used in order to follow up the response of treatment in psoriasis patients.

Systemic therapies, such as biological or conventional systemic therapy, allow a decrease in the inflammatory activity of psoriasis. These results correlate with the scientific data, which support the beneficial effect of biological therapy in decreasing inflammation in psoriasis $(20,21)$. Coimbra and Santos-Silva (22) recommend CRP as the most sensitive biomarker for severity evaluation and monitoring of treatment response in psoriasis patients. Gokalp (23) also suggested that CRP is a marker not only for psoriasis activity but also for systemic inflammation.

By evaluating the blood levels of CRP, ESR and fibrinogen, according to the treatment the therapeutic efficacy of the treatment was demonstrated using acute phase reactants, such as ESR, CRP and fibrinogen.

Imaging techniques are also used to assess non-invasive skin changes in psoriasis or high performance liquid chromatography or cell membrane fluidity; however, this study aimed to evaluate only the inflammatory activity (24-27).

Because psoriasis is a chronic condition, it is also necessary to evaluate patients at the time of maximum symptoms and consider evaluating its comorbidities.

In conclusion, this study illustrates a strong correlation between plasmatic levels of acute phase reactants, such as CRP, ESR, fibrinogen and the inflammatory activity of psoriasis. By using these inflammatory markers, it was concluded that the best results in reducing inflammatory activity were achieved by biological therapy, which induces a decrease in blood levels of CRP, ESR and fibrinogen. In addition, it indirectly points to a reduction of complications associated with the inflammatory syndrome that represents the basis of this disease. The authors propose CRP, ESR and fibrinogen as prognostic indicators of the unfavorable evolution of psoriasis. It is also less expensive to evaluate these three markers in comparison with others and their determination is easier.

\section{Acknowledgements}

Professional editing, linguistic and technical assistance performed by Irina Radu, Individual Service Provider, certified translator in Medicine and Pharmacy (certificate credentials: series E no. 0048).

\section{Funding}

No funding was received.

\section{Availability of data and materials}

All data and materials supporting the results of the present study are available in the published article.

\section{Authors' contributions}

DV, AIP and AIG contributed to the acquisition of data. All authors contributed to the critical revision of manuscript for important intellectual content. All authors contributed to the acquisition of the data. CG, LGS, EPA were responsible for the research design. CG, LGS and EPA were involved in writing the manuscript. CG carried out the statistical analysis. All authors read and approved the final version of the manuscript.

\section{Ethics approval and consent to participate}

This study was approved by the Clinical Research Ethics Committee of 'Sf. Spiridon' Clinical Emergency County Hospital (Iasi, Romania) and the Ethics Committee of 'Gr. T. Popa' University of Medicine and Pharmacy (Iasi, Romania). Written informed consents were obtained by the patients and/or guardians.

\section{Patient consent for publication}

Written informed consent was obtained from all patients prior to publication.

\section{Competing interests}

The authors declare that they have no competing interests.

\section{References}

1. Lin $X$ and Huang T: Co-signaling molecules in psoriasis pathogenesis: Implications for targeted therapy. Hum Immunol 76: 95-101, 2015.

2. Ghosh A and Panda S: Recent understanding of the etiopathogenesis of psoriasis. Indian J Paediatr Dermatol 18: 1-8, 2017

3. Caruntu C, Boda D, Dumitrascu G, Constantin C and Neagu M Proteomics focusing on immune markers in psoriatic arthritis. Biomarkers Med 9: 513-528, 2015.

4. Căruntu C, Boda D, Musat S, Căruntu A and Mandache E: Stress-induced mast cell activation in glabrous and hairy skin. Mediators Inflamm 2014: 105950, 2014.

5. Farshchian M, Ansar A, Sobhan M and Hoseinpoor V: C-reactive protein serum level in patients with psoriasis before and after treatment with narrow-band ultraviolet B. An Bras Dermatol 91: 580-583, 2016.

6. Lowes MA, Suárez-Fariñas M and Krueger JG: Immunology of psoriasis. Annu Rev Immunol 32: 227-255, 2014.

7. Li R, Wang J, Wang X, Zhou J, Wang M, Ma H and Xiao S: Increased $\beta \operatorname{TrCP}$ are associated with imiquimod-induced psoriasis-like skin inflammation in mice via NF- $\mathrm{KB}$ signaling pathway. Gene 592: 164-171, 2016.

8. Pepys MB and Hirschfield GM: C-reactive protein: A critical update. J Clin Invest 111: 1805-1812, 2003.

9. Rocha-Pereira P, Santos-Silva A, Rebelo I, Figueiredo A, Quintanilha A and Teixeira F: The inflammatory response in mild and in severe psoriasis. Br J Dermatol 150: 917-928, 2004.

10. Gisondi P, Lora V, Bonauguri C, Russo A, Lippi G and Girolomoni G: Serum chemerin is increased in patients with chronic plaque psoriasis and normalizes following treatment with infliximab. Br J Dermatol 168: 749-755, 2013.

11. Qu D, Liu J, Lau CW and Huang Y: IL-6 in diabetes and cardiovascular complications. Br J Pharmacol 171: 3595-3603, 2014.

12. Devaraj S, Valleggi S, Siegel D and Jialal I: Role of C-reactive protein in contributing to increased cardiovascular risk in metabolic syndrome. Curr Atheroscler Rep 12: 110-118, 2010

13. Biljan D, Situm M, Kostović K, Batinac T and Matisić D: Acute phase proteins in psoriasis. Coll Antropol 33: 83-86, 2009.

14. Isha, Jain VK and Lal H: C-reactive protein and uric acid levels in patients with psoriasis. Indian J Clin Biochem 26: 309-311, 2011.

15. Negrei C, Ginghină O, Căruntu C, Burcea Dragomiroiu GT, Jinescu $G$ and Boda D: Investigation relevance of methotrexate polyglutamates in biological systems by high performance liquid chromatography. Rev Chim 66: 766-768, 2015.

16. Boda D, Negrei C, Nicolescu F and Balalau C: Assessment of some oxidative stress parameters in methotrexate treated psoriasis patients. Farmacia 62: 704-710, 2014. 
17. Zamanian A, Houshang Ehsani A, Bahareh Darvari S, Mehran G and Azizpour A: Trend of C-reactive protein anderythrocyte sedimentation rates in psoriatic patients on treatment of standard protocol of infliximab. GMJ 4: 8-13, 2015.

18. Strober B, Teller C, Yamauchi P, Miller JL, Hooper M, Yang YC and Dann F: Effects of etanercept on C-reactive protein levels in psoriasis and psoriatic arthritis. Br J Dermatol 159: 322-330, 2008.

19. Kanelleas A, Liapi C, Katoulis A, Stavropoulos P, Avgerinou G, Georgala S, Economopoulos T, Stavrianeas NG and Katsambas A: The role of inflammatory markers in assessing disease severity and response to treatment in patients with psoriasis treated with etanercept. Clin Exp Dermatol 36: 845-850, 2011.

20. Olteanu R, Constantin MM,Zota A, Dorobantu DM, Constantin T, Serban ED, Balanescu P, Mihele D and Gheuca-Solovastru L: Original clinical experience and approach to treatment study with interleukine $12 / 23$ inhibitor in moderate-to-severe psoriasis patients. Farmacia 64: 918-921, 2016.

21. Olteanu R, Zota A and Constantin M: Biosimilars: An update on clinical trials (review of published and ongoing studies). Acta Dermatovenerol Croat 25: 57-66, 2017.

22. Coimbra S and Santos-Silva A: Biomarkers of psoriasis severity and therapy monitoring. World J Dermatol 3: 15-27, 2014
23. Gocalp H: Effect of psoriasis severity on inflammation parameters: Controlled study. Turk Arch Dermatol Venereol 52: 91-94, 2018

24. Căruntu C, Boda D, Căruntu A, Rotaru M, Baderca F and Zurac S: In vivo imaging techniques for psoriatic lesions. Rom J Morphol Embryol 55 (Suppl 3): 1191-1196, 2014.

25. Ilie MA, Caruntu C, Lixandru D, Tampa M, Georgescu SR, Constantin MM, Constantin C, Neagu M, Zurac SA and Boda D: In vivo confocal laser scanning microscopy imaging of skin inflammation: Clinical applications and research directions. Exp Ther Med 17: 1004-1011, 2019.

26. Negrei C, Caruntu C, Ginghina O, Dragomiroiu GT, Toderescu CD and Boda D: Qualitative and quantitative determination of methotrexate polyglutamates in erythrocytes by high performance liquid chromatography. Rev Chim 66: 607-610, 2015.

27. Negrei C, Arsene AL, Toderescu CD, Boda D and Ilie M: Acitretin treatment in psoriasis may influence the cell membrane fluidity. Farmacia 60: 767-771, 2012.

This work is licensed under a Creative Common Attribution-NonCommercial-NoDerivatives 4.0 International (CC BY-NC-ND 4.0) License. 